\title{
Electron Absorption Spectrum of Cobalt (II) -Doped Trisphenanthrolinezinc Nitrate Dihydrate
}

\author{
Curt W. Reimann \\ Institute for Materials Research, National Bureau of Standards, Washington, D.C. 20234
}

(May 10, 1966)

\begin{abstract}
The electron absorption spectrum of cobalt(II)-doped trisphenanthrolinezinc nitrate dihydrate has been observed at room temperature and at $77^{\circ} \mathrm{K}$. Broad bands appear at 10,750 and 22,000 $\mathrm{cm}^{-1}$ which are assigned to the ${ }^{4} \mathrm{~T}_{1 g}(\mathrm{~F}) \longleftrightarrow \rightarrow{ }^{4} \mathrm{~T}_{2 g}(\mathrm{~F})$ and the ${ }^{4} \mathrm{~T}_{1 g}(\mathrm{~F}) \leftarrow \rightarrow{ }^{4} \mathrm{~T}_{1 g}(\mathrm{P})$ transitions respectively. A broad weak band occurs near $19,000 \mathrm{~cm}^{-1}$ but at $77^{\circ} \mathrm{K}$ this band is resolved into a series of narrower bands. These bands are assigned to ${ }^{2} \mathrm{G}$ transitions. The relationship between the spectrum of cobalt(II) trisphenanthrolinezinc nitrate dihydrate and the solution spectra of cobalt(II) in ammonia and ethylenediamine is discussed.

Key Words: Trisphenanthroline cobalt(II) nitrate dihydrate, trisphenanthrolinezinc nitrate dihydrate, electron absorption spectrum.
\end{abstract}

\section{Introduction}

Several spectroscopic studies of single crystals of octahedrally coordinated cobalt(II) have appeared recently [1-4]. ${ }^{1}$ Cobalt(II) spectra are particularly interesting in that they are characterized by narrow spin-forbidden bands as well as by broad spin-allowed bands which are more typical of $d-d$ transitions. In each of the previously reported spectra, however, the ligand field strength parameter, D $q$, was less than 1000 . Such $\mathrm{D} q$ values are smaller than would be expected in octahedral cobalt(II) complexes of ammonia, ethylenediamine or 1, 10 phenanthroline. These complexes assume some importance since Liehr [5] has constructed a diagram of electron energy versus ligand field strength $\mathrm{D} q$ for the $d^{7}$ system which indicates that for $\mathrm{D} q$ greater than about 1100 the ${ }^{4} \mathrm{~A}_{2 g}(\mathrm{~F})$ state lies higher than the ${ }^{4} \mathrm{~T}_{1 g}(\mathrm{P})$ state while at lower $\mathrm{D} q$ the reverse obtains. Ballhausen and Jorgensen [6] reported the spectra of cobalt(II) in ammonia and ethylenediamine solutions which quite clearly correspond to $\mathrm{D} q$ greater than 1000 . These workers assigned weak broad bands near $19,000 \mathrm{~cm}^{-1}$ as ${ }^{4} \mathrm{~T}_{1 g}(\mathrm{~F}) \leftarrow \rightarrow{ }^{4} \mathrm{~A}_{2 g}(\mathrm{~F})$ transitions and bands near 21,000 $\mathrm{cm}^{-1}$ as arising from ${ }^{4} \mathrm{~T}_{1 g}(\mathrm{~F}) \longleftrightarrow{ }^{4} \mathrm{~T}_{1 g}(\mathrm{P})$ transitions thereby suggesting that the ${ }^{4} \mathrm{~A}_{2 g}(\mathrm{~F})$ state lies lower than the ${ }^{4} \mathrm{~T}_{1 g}(\mathrm{P})$ state in each of their spectra.

Exploratory studies were carried out on alcohol solutions of cobalt (II) and 1, 10 phenanthroline and they revealed the following:

(1) The ligand field strength provided by 1, 10 phenanthroline as evidenced by spectral shifts was greater than that provided by either ammonia or ethylenediamine.

${ }^{1}$ Figures in brackets indicate the literature references at the end of this paper.
(2) a weak broad band near $19,000 \mathrm{~cm}^{-1}$ was present as it had been in ammonia and ethylenediamine solutions of cobalt (II).

In an attempt to bring new evidence to bear on possible assignments single crystals of cobalt(II)-doped trisphenanthrolinezinc nitrate dihydrate were grown and low-temperature spectra were investigated. In what follows the outcome of this investigation is described.

\section{Experimental Procedure}

Crystals of cobalt(II)-doped trisphenanthrolinezinc nitrate dihydrate were grown from ethyl alcohol solutions of zinc nitrate, cobalt(II) nitrate and phenanthroline in slight excess of the stoichiometrically required amount. Crystals of trisphenanthrolinezinc nitrate dihydrate are triclinic with two molecules per unit cell [7]. Polarized spectra were measured on a spectrophotometer (Cary 14) with Glan-Thompson polarizers. Spectra at $77^{\circ} \mathrm{K}$ were taken with single crystals mounted on brass disks in thermal contact with the coolant which was contained in a simple Dewar. When viewed with polarized light crystals of cobalt(II)-doped trisphenanthrolinezinc nitrate dihydrate vary in color from bright orange to pale yellow.

\section{Results}

The absorption spectrum of cobalt(II)-doped trisphenanthrolinezinc nitrate dihydrate from 8,000 to $20,000 \mathrm{~cm}^{-1}$ is shown in figure 1 . The features of this spectrum are identical with the alcohol-water solution spectrum of cobalt(II) nitrate and phenanthroline and very similar to the solution spectra of $\mathrm{Co}\left(\mathrm{NH}_{3}\right)_{6}^{++}$and 


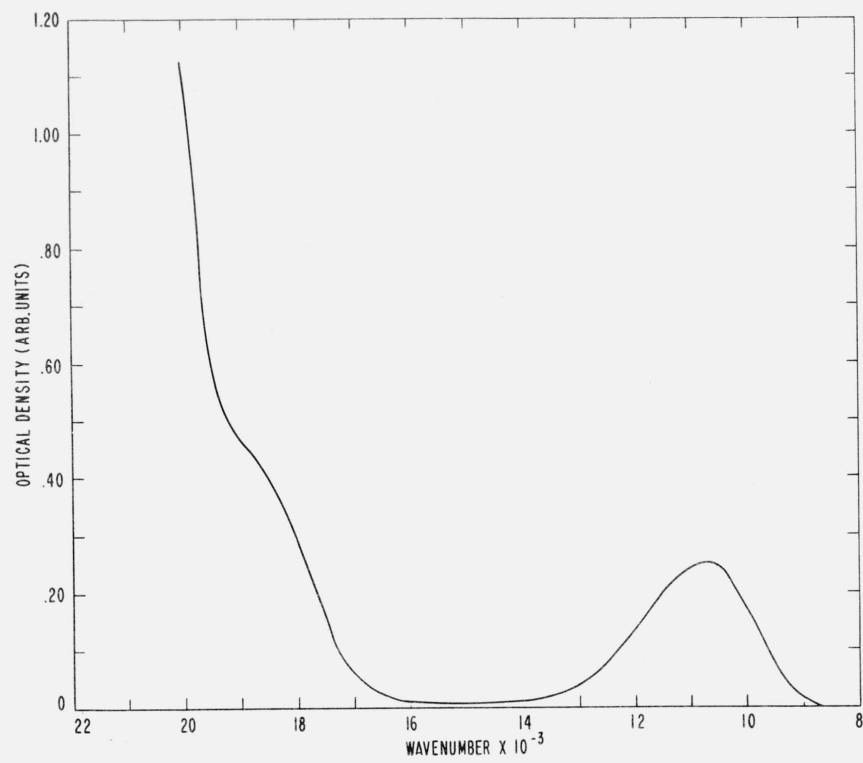

FIGURE 1. Single crystal spectrum of cobalt (II)-doped trisphenanthrolinezinc nitrate dihydrate from 8000 to $20,000 \mathrm{~cm}^{-1}$

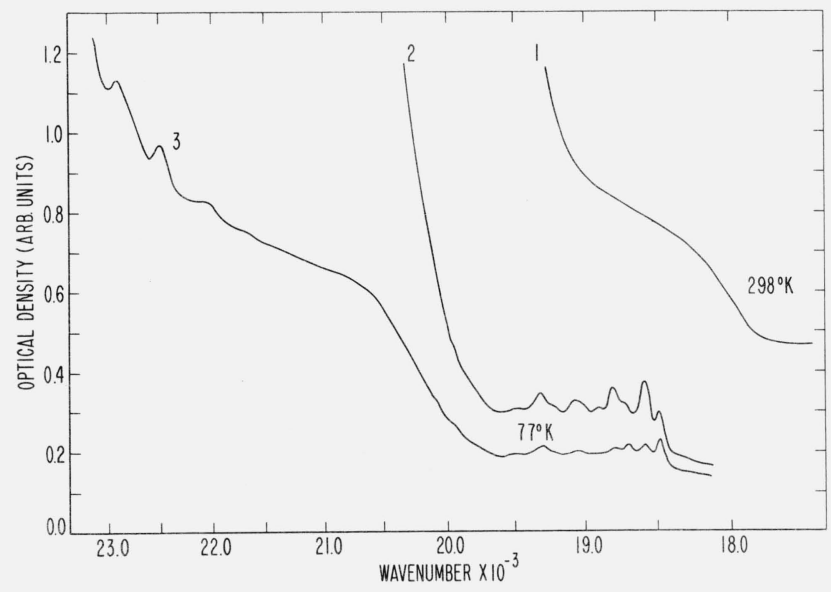

FIGURE 2. Single crystal spectra of cobalt (II)-doped trisphenanthrolinezinc nitrate dihydrate near $19,000 \mathrm{~cm}^{-1}$.

Curve 1 shows the spectrum at room temperature while curves 2 and 3 are polarized spectra taken at $77^{\circ} \mathrm{K}$. Polarized spectra were taken along the direction in which the narrow band system was best resolved (curve 2) and perpendicular to this direction.

$\mathrm{Co}(\mathrm{en})_{3}^{++}$reported by Ballhausen and Jorgensen. For purposes of calculating $\mathrm{D}_{q}$ and $\mathrm{B}$ for $\mathrm{Co}(\text { phen })_{3}^{++}$and comparing the results with those obtained by Ballhausen and Jorgensen for $\mathrm{Co}\left(\mathrm{NH}_{3}\right)_{6}^{++}$and $\mathrm{Co}(\mathrm{en})_{3}^{++}$ spectral data were obtained from solutions of cobalt(II) nitrate and phenanthroline. To obtain the best values for the band energies samples were prepared from carefully degassed solutions. Absorption bands were observed in these solutions at $10,750 \mathrm{~cm}^{-1}, 22,000$ $\mathrm{cm}^{-1}$ and a broad weak band occurred at about 19,000 $\mathrm{cm}^{-1}$.
At $77^{\circ} \mathrm{K}$ the absorption bands in cobalt(II) doped trisphenanthrolinezinc nitrate dihydrate narrowed slightly and relatively sharp bands appeared in the region from $18,450 \mathrm{~cm}$ to $23,000 \mathrm{~cm}^{-1}$ (fig. 2). The narrow bands from $18,450 \mathrm{~cm}$ to $19,800 \mathrm{~cm}^{-1}$ were polarized and most clearly resolved along the direction in which the crystal appeared orange.

\section{Discussion}

Cobalt (II), a $d^{7}$ system, has free ion states ${ }^{4} \mathrm{~F},{ }^{4} \mathrm{P}$, ${ }^{2} \mathrm{P},{ }^{2} \mathrm{D},{ }^{2} \mathrm{G},{ }^{2} \mathrm{H}$, and ${ }^{2} \mathrm{~F}$. In an octahedral crystalline field the lowest free ion state, ${ }^{4} \mathrm{~F}$, splits into two orbital triplets, ${ }^{4} \mathrm{~T}_{1 g}$ and ${ }^{4} \mathrm{~T}_{2 g}$, and an orbital singlet, ${ }^{4} \mathrm{~A}_{2 g}$, while the next lowest free ion state, ${ }^{4} \mathrm{P}$, remains unsplit $\left({ }^{4} \mathrm{~T}_{1 g}\right)$. Transitions from the ground state, ${ }^{4} \mathrm{~T}_{1 g}(\mathrm{~F})$, to ${ }^{4} \mathrm{~T}_{2 g}(\mathrm{~F}),{ }^{4} \mathrm{~A}_{2 g}(\mathrm{~F})$ and ${ }^{4} \mathrm{~T}_{1 g}(\mathrm{P})$ lead to broad banded absorptions in the visible and near infrared spectral regions. Typically, the spectrum of cobalt (II) in an octahedral environment consists of a broad band in the near infrared $\left({ }^{4} \mathrm{~T}_{1 g}(\mathbf{F}) \leftarrow \rightarrow{ }^{4} \mathrm{~T}_{2 g}(\mathbf{F})\right)$, a broad and less intense band in the visible $\left({ }^{4} \mathrm{~T}_{1 g}(\mathrm{~F})\right.$ $\left.\leftarrow \rightarrow{ }^{4} A_{2 g}(F)\right)$ and another broad band in the visible

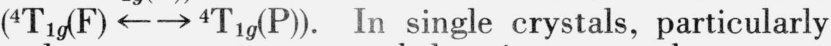
at low temperatures, much less intense and narrower bands appear in the spectrum of cobalt (II). These bands are usually assigned to spin-forbidden transitions to doublet states. The spectrum of cobalt (II) doped trisphenanthrolinezinc nitrate dihydrate will now be considered in terms of the simple octahedral picture.

The band at $10,750 \mathrm{~cm}^{-1}$ is assigned to the ${ }^{4} \mathrm{~T}_{1 g}(\mathrm{~F})$ $\leftarrow \rightarrow{ }^{4} \mathrm{~T}_{2 g}(\mathrm{~F})$ transition and the broad absorption located at $22,000 \mathrm{~cm}^{-1}$ is assigned as the ${ }^{4} \mathrm{~T}_{1 g}(\mathrm{~F})$ $\leftarrow \rightarrow{ }^{4} \mathrm{~T}_{1 g}(\mathrm{P})$ transition. The assignment of the ${ }^{4} \mathrm{~T}_{1 g}(\mathrm{P})$ and the ${ }^{4} \mathrm{~T}_{2 g}(\mathrm{~F})$ levels permits the calculation of $\mathrm{D}_{q}$ and $\mathrm{B}$ by the method of Tanabe and Sugano [8]. $\mathrm{D}_{q}$ is the crystal field strength parameter and $\mathrm{B}$ is the interelectronic repulsion parameter. From the computed values of $\mathrm{D}_{q}$ and $\mathrm{B}$ the energy of the ${ }^{4} \mathrm{~A}_{2 g}$ level is found to be about $22,800 \mathrm{~cm}^{-1}$ above the ${ }^{4} \mathrm{~T}_{1 g}(\mathrm{~F})$ ground state. From the assignments of the ${ }^{4} \mathrm{~T}_{1 g}(\mathrm{P})$ and ${ }^{4} \mathrm{~T}_{2 g}(\mathrm{~F})$ states in $\mathrm{Co}\left(\mathrm{NH}_{3}\right)_{6}^{++}$and $\mathrm{Co}(\mathrm{en})_{3}^{++}$made by Ballhausen and Jorgensen the $\mathrm{D}_{q}$ and $\mathrm{B}$ values can be computed and these values utilized, in turn, to calculate the ${ }^{4} \mathrm{~A}_{2 g}(\mathrm{~F})$ levels. The calculated $\mathrm{D}_{q}, \mathrm{~B}$, and ${ }^{4} \mathrm{~A}_{2 g}(\mathrm{~F})$ levels for $\mathrm{Co}(\text { phen })_{3}^{++}, \mathrm{Co}\left(\mathrm{NH}_{3}\right)_{6}^{++}$and $\mathrm{Co}(\mathrm{en})_{3}^{++}$ are presented in Table 1.

TABLE 1

\begin{tabular}{|c|c|c|c|}
\hline & $\mathrm{D} q$ & B & ${ }^{4} \mathbf{A}_{2 g}$ \\
\hline Co(phen) ${ }_{3}^{++}$ & 1206 & 837 & 22813 \\
\hline $\mathrm{Co}\left(\mathrm{NH}_{3}\right)_{6}^{++}$ & 1023 & 889 & 19228 \\
\hline Co(en) $)_{3}^{++}$ & 1102 & 875 & 20818 \\
\hline
\end{tabular}


In addition to the assignment of the ${ }^{4} \mathrm{~T}_{1 g}(\mathrm{P})$ and ${ }^{4} \mathrm{~T}_{2 g}(\mathrm{~F})$ states Ballhausen and Jorgensen attribuied weak absorption near $19,000 \mathrm{~cm}^{-1}$ in their spectra to the ${ }^{4} \mathrm{~T}_{1 g}(\mathrm{~F}) \leftarrow \rightarrow{ }^{4} \mathrm{~A}_{2 g}(\mathrm{~F})$ transition. It is clear, however, that such an assignment in Co(phen) ${ }_{3}^{++}$is less tenable not only because the absorption near $19,000 \mathrm{~cm}^{-1}$ is resolvable at low temperatures into a series of sharp bands but also because the computed separation of the ${ }^{4} A_{2 g}(F)$ level from the ground state is $22,800 \mathrm{~cm}^{-1}$. Our interpretation of the spectrum of $\mathrm{Co}(\text { phen })_{3}^{++}$is based upon the evidence that the absorption near $19,000 \mathrm{~cm}^{-1}$ can be assigned as transitions to other states within the cobalt (II) manifold. In terms of this interpretation the ${ }^{4} \mathrm{~T}_{1 g}(\mathrm{~F}) \leftarrow \rightarrow{ }^{4} \mathrm{~A}_{2 g}(\mathrm{~F})$ band lies above $20,000 \mathrm{~cm}^{-1}$ but must remain unspecified because of the more intense ${ }^{4} \mathrm{~T}_{1 g}(\mathrm{~F}) \longleftrightarrow \rightarrow{ }^{4} \mathrm{~T}_{1 g}(\mathrm{P})$ and charge transfer bands which occur in this region.

The narrow bands observed near $19,000 \mathrm{~cm}^{-1}$ are analogous to those reported by Ferguson and by Ferguson, Wood, and Knox who assigned the bands to transitions to doublet levels. Liehr has constructed an energy level versus ligand field strength, $\mathrm{D}_{q}$, diagram for the $d^{7}$ electronic system in octahedral symmetry. Ferguson, Wood, and Knox have computed the energies of the doublet states for cobalt(II) in $\mathrm{KCOF}_{3}, \mathrm{CoCl}_{2}, \mathrm{CoBr}_{2}$, and $\mathrm{CoWO}_{4}$ utilizing the $\mathrm{D}_{q}$ and $B$ values obtained from their observed spectra. Briefly, these workers show that in the free ion the ${ }^{2} \mathrm{G}$ states lie lowest of the doublet states with ${ }^{2} \mathrm{P}$ and ${ }^{2} \mathrm{H}$ levels occurring some $4,000 \mathrm{~cm}^{-1}$ above ${ }^{2} \mathrm{G} .{ }^{2} \mathrm{~F}$ and ${ }^{2} \mathrm{D}$ lie still higher. In an octahedral crystalline field the ${ }^{2} \mathrm{G}$ states split into six levels four of which are relatively insensitive to the strength of the ligand field. The energies of the other two states arising from ${ }^{2} \mathrm{G}$ show a rather strong dependence upon the ligand field strength. One of these states increases while the other decreases in energy with increasing ligand field strength. The energies of states arising from ${ }^{2} \mathrm{H},{ }^{2} \mathrm{P}$, ${ }^{2} \mathrm{D}$, and ${ }^{2} \mathrm{~F}$ of the free ion increase moderately rapidly with increasing ligand field strength. This analysis shows that transitions to four ${ }^{2} \mathrm{G}$ states would be expected to give rise to transitions in a spectral region which is free of transitions to other doublet states. More specifically, one would expect that transition to the four intermediate ${ }^{2} \mathrm{G}$ states would give rise to $\mathrm{ab}$ sorption in the 15,000 to $20,000 \mathrm{~cm}^{-1}$ region and that the lowest and highest of this set of ${ }^{2} \mathrm{G}$ states would be separated by about $1000 \mathrm{~cm}^{-1}$. In the spectrum of cobalt(II) doped trisphenanthrolinezinc nitrate dihydrate narrow bands are observed from $18,450 \mathrm{~cm}^{-1}$ to $19,500 \mathrm{~cm}^{-1}$ and these are assigned to transitions to the ${ }^{2} \mathrm{G}$ states.

Figure 2 shows, however, that nine peaks are resolved between 18,450 and 19,500 $\mathrm{cm}^{-1}$. The number of observed bands cannot be accounted for on the basis of symmetry lower than octahedral since reduction in symmetry could produce only two additional bands.
Moreover, the occurrence of too many bands cannot be accounted for on the basis of transitions from a thermally populated excited state. The latter conclusion follows from the fact that the minimum separation between observed bands is about $100 \mathrm{~cm}^{-1}$ and no bands were observed to diminish as the temperature was reduced from 77 to $20^{\circ} \mathrm{K}$. The occurrence of more peaks than the number of ${ }^{2} \mathrm{G}$ levels is undoubtedly associated with the fact that the bands in question are strictly forbidden in octahedral symmetry and arise via a coupling to antisymmetric vibrations. That is, the electronic transitions must be accompanied by a simultaneous change of one quantum in an antisymmetric mode. In principle progressions of symmetric vibrational modes can be superimposed on the perturbing antisymmetric mode to greatly complicate the appearance of an electronic absorption band. It appears therefore that the vibrational structure which was so evident in the cobalt(II) crystal spectra of Ferguson and Ferguson, Wood, and Knox also arises in the spectrum of cobalt(II)-doped trisphenanthrolinezinc nitrate dihydrate.

While no conclusions regarding the bands near $19,000 \mathrm{~cm}^{-1}$ in $\operatorname{Co}\left(\mathrm{NH}_{3}\right)_{6}^{++}$and in $\operatorname{Co}(\mathrm{en})_{3}^{++}$should be drawn without low temperature spectra of the corresponding crystalline materials it appears likely that these bands are analogous to those which we have assigned to ${ }^{2} \mathrm{G}$ transitions. The closeness in the $\mathrm{D} q$ and $B$ values in these materials and the insensitivity of the energy of the intermediate ${ }^{2} \mathrm{G}$ levels to small changes in $\mathrm{D} q$, support this notion.

The author thanks Harry C. Allen, Jr., Deputy Director of the Institute for Materials Research for suggesting that this investigation be carried out.

\section{References}

[1] J. Ferguson, D. L. Wood, and K. Knox, J. Chem. Phys. 39, 881 (1963).

[2] J. Ferguson, J. Chem. Phys. 32, 533 (1960).

[3] R. Pappalardo, D. L. Wood and R. C. Linares, Jr., J. Chem. Phys. 35, 2041 (1961).

[4] W. Low, Phys. Rev. 109, 256 (1958).

[5] A. D. Liehr, J. Phys. Chem. 67, 1314 (1963).

[6] C. J. Ballhausen and C. K. Jorgensen, Acta Chem. Scand. 9, 397 (1955).

[7] G. F. Kokoszka, C. W. Reimann, H. C. Allen, Jr., and G. Gordon (in preparation)

[8] Y. Tanabe and S. Sugano, J. Chem. Soc. Japan 9, 753 (1954).

(Paper 70A5-413)

(Paper 70A5-413) 